\title{
DFT-Based OFDMA System with Phase Modulation for Broadband Communication
}

\author{
Farouk Abduh Kamil Al-Fuhaidy ${ }^{*}$, Faisal Saif Alkamali", Khaled Abdullah Al-Soufy ${ }^{*}$ \\ Department of Electrical Engineering, Ibb University, Ibb, Yemen \\ Email address: \\ farouqakh@gmail.com (F. A. K. Al-Fuhaidy), faisalalkamali@yahoo.com (F. S. Alkamali), kalsoufi@gmail.com (K. A. Al-Soufy) \\ ${ }^{*}$ Corresponding author
}

\section{To cite this article:}

Farouk Abduh Kamil Al-Fuhaidy, Faisal Saif Alkamali, Khaled Abdullah Al-Soufy. DFT-Based OFDMA System with Phase Modulation for Broadband Communication. American Journal of Electrical and Computer Engineering. Vol. 3, No. 1, 2019, pp. 1-9.

doi: 10.11648/j.ajece.20190301.11

Received: April 10, 2019; Accepted: May 20, 2019; Published: June 13, 2019

\begin{abstract}
This paper proposes, a DFT-based OFDMA with phase modulation (DFT-OFDMA-PM) system. One main advantage of the phase modulated system is the constant envelope (CE) resulted signal, i.e, $0 d B P A P R$, the second advantage is the ability to improve the diversity of multipath channels. These advantages have been exploited in the proposed system of this paper. The performance of the proposed system in terms of the bit error rate (BER) is studied and investigated and compared to the previously proposed DCT-OFDMA-PM system and the conventional OFDMA system without PM via computer simulation. The key parameter that affects the performance of the PM systems, the modulation index, is also studied and the optimum value is selected using exhaustive simulation scenarios. Moreover, the PAPR is also simulated for the proposed system and compared to the conventional system. Simulation results show the significant improvement of the proposed system in terms of PAPR and BER when compared to the conventional system. The simulation results for the proposed system show the effectiveness of the proposed system for wireless broadband communications.
\end{abstract}

Keywords: DFT, DCT, OFDMA, PAPR, Phase Modulation

\section{Introduction}

Wireless digital communication is rapidly expanding, resulting in a demand for wireless systems that are reliable and have a high spectral efficiency [1]. Orthogonal frequency division multiple access (OFDMA) is an attractive technology to deal with the detrimental effects of multipath fading, but it faces several inherent disadvantages such as the high peak-to-average-power ratio (PAPR) and the sensitivity to carrier frequency offsets $[2,3]$. In cellular applications, a big advantage of OFDMA is its robustness in the presence of multipath signal propagation [4]. The immunity to multipath derives from the fact that an OFDMA system transmits information on $M$ orthogonal frequency carriers, each operating at $1 / M$ times the bit rate of the information signal. On the other hand, the OFDMA waveform exhibits very pronounced envelope fluctuations resulting in a PAPR. Signals with a high PAPR require highly linear power amplifiers to avoid excessive intermodulation distortion. To achieve this linearity, the amplifiers have to operate with a large backoff from their peak power. The result is low power efficiency (measured by the ratio of transmitted power to dc power dissipated), which places a significant burden on portable wireless terminals [5]. Several PAPR reductions techniques for multicarrier transmissions are surveyed by $\mathrm{S}$. H. Han [6]. These techniques can be classified into two groups, the first one which is called distortion reduction techniques, such as, windowing and clipping [7]. The second one called non distortion reduction techniques, which can be classified into two different types. Which are either based on multiple signal representations, such as, the partial transmit sequence (PTS) technique, the selective mapping (SLM) technique, and the interleaving technique, or technique based on signal transformation such as phase modulation (PM) $[5$, 6, 8-12]. The PM based systems have several advantages. The resulted signal by the PM-based system has a constant envelope (i.e. $0 \mathrm{~dB}$ PAPR) which allows the PA to operate near the saturation region, thus maximizing the power efficiency. Therefore, these systems have a larger coverage 
area since more signal power is radiated into the channel. The goal of this paper is to propose a DFT-based OFDMA with $\mathrm{PM}$ and comparing its performance with the recently proposed DCT-based OFDMA (DCT-OFDMA-PM) system by F. AL-Fuhaidy [5]. Although, the key parameter affecting the PM-based system, the modulation index, is also studied and the optimum value is chosen using simulation which verified the mathematical indication. PM-based systems also have the ability to improve the diversity of multipath channels which is studied and verified using simulation of bit error rate (BER).

The reminder of this paper is organized as follows. Section 2 presents an overview on the conventional OFDMA system. Then, the proposed DFT-OFDMA-PM system model is derived and introduced in Section 3, the recently proposed DCT-OFDMA-PM system is also presented and a comparison study between the DFT-OFDMA-PM and the DCT-OFDMA-PM systems is discussed in case of the type of orthogonal subcarriers used by each system. Simulation results for the proposed system DFT-OFDMA-PM in terms of bit error rate (BER), modulation index, $h$, and the PAPR, are presented, discussed, and compared with the DCT-
OFDMA-PM and the conventional OFDMA systems by Section 4. Finally, Section 5 concludes the paper.

\section{The Traditional OFDMA System}

The OFDMA system that is used for mobile communications was first proposed by R. Nogueroles [13]. Figure 1 presents a block diagram for the conventional DFTbased OFDMA system. It is based on multicarrier FDMA, where each user is assigned to a set of randomly selected subchannels. There are $U$ uplink users communicating with a base station through independent multipath fading channels. A total of $M$ subcarriers are assumed and each user is assigned $N$ subcarriers. At the transmitter, the encoded signals are modulated and then the subcarriers are mapped in the frequency domain. After that, the IDFT is performed, and a cyclic prefix (CP) is added to the resulting signal. Finally, the resulting signal is transmitted through the wireless channel. At the receiver, the CP is removed and the DFT is then applied. Finally, the subcarrier remapping, the equalization, the demodulation and the decoding operations are performed.

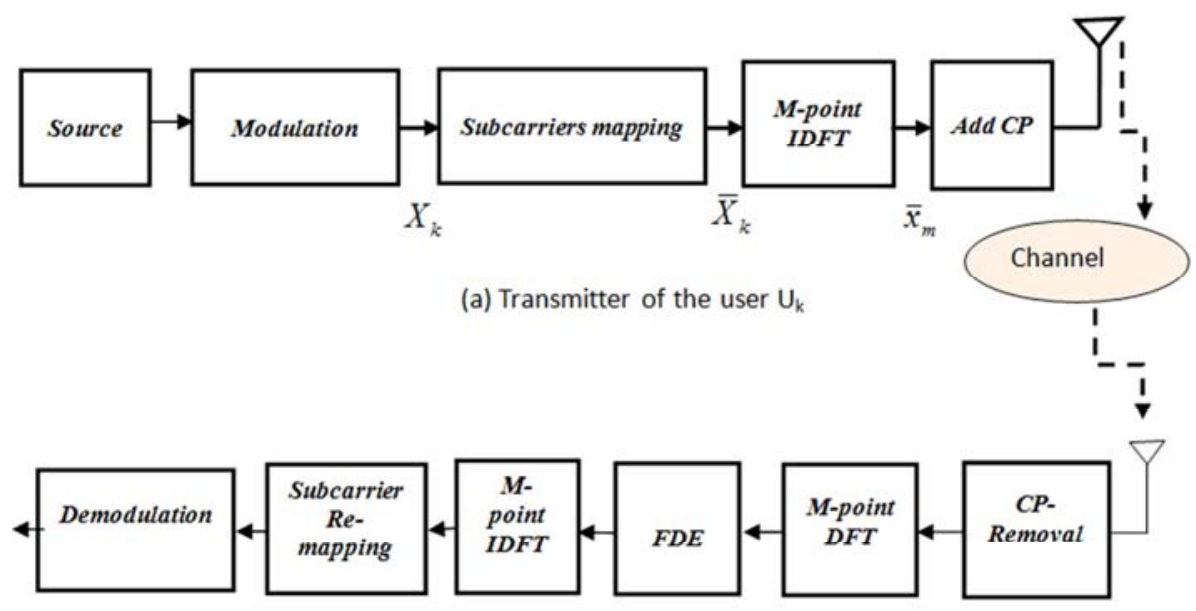

(b) Receiver of the user $U_{k}$

Figure 1. A block diagram for the DFT OFDMA system over frequency selective fading channel.

\section{The OFDMA Proposed Systems}

This section firstly presents the proposed DFT-based OFDMA system with phase modulation along with its mathematical model. Then, a brief literature on the recently proposed DCT-based OFDMA system with phase modulation is introduced in order to illustrate the similarity and differences to the proposed system.

\subsection{The Proposed DFT-OFDMA-PM System}

This section introduces the proposed system named DFTOFDMA-PM, which refers to Discrete Fourier Transformbased Orthogonal Frequency Division Multiple Access with Phase Modulation. The proposed system is suggested as a new transceiver scheme for the OFDMA system. The block diagram which illustrates the proposed system is shown in
Figure 2. Now, an illustration of the proposed system mathematical model is presented.

During each block interval, T-seconds, an M-point IDFT calculates a block of time samples $x(n)$. Next step, a high PAPR DFT-OFDMA sequence, $x(n)$, is passed through a phase modulator to obtain a $0 \mathrm{~dB}$ PAPR sequence $s(n)=\exp$ (j $C x(n))$, where $C$ is a scaling constant. Then $N_{g}$ samples of $\mathrm{CP}$ are added to $s(n)$. The continuous-time DFT-OFDMAPM signal, $s(t)$, is then generated at the output of the digitalto-analog (D/ A) converter. This baseband signal can be expressed as follows $[5,9,13]$ :

$$
S(t)=A e^{j \emptyset(t)}=A e^{j[2 \pi h \cdot m(t)+\theta]}, T_{g} \leq t<T
$$

where $A$ is the signal amplitude, $h$ is the modulation index, $\theta$ is an arbitrary phase offset used to achieve phase continuous modulation $[5,13] . T_{g}$ is the guard period, $T$ is the block 
period, and $m(t)$ is a real-valued DFT-OFDMA signal and given as:

$$
m(t)=C_{N} \sum_{k=1}^{M} X_{k} q_{k}(t)
$$

where $C_{N}$ is the normalization constant used to normalize the variance of the message signal $\left(\sigma_{x}^{2}=1\right)$ after subcarrier mapping and consequently the variance of the phase signal, $\sigma_{\varnothing}^{2}=(2 \pi h)^{2}$. This requirement is achieved by setting $C_{N}$ as follows:

$$
C_{N}=\sqrt{\frac{2}{M \sigma_{x}^{2}}}
$$

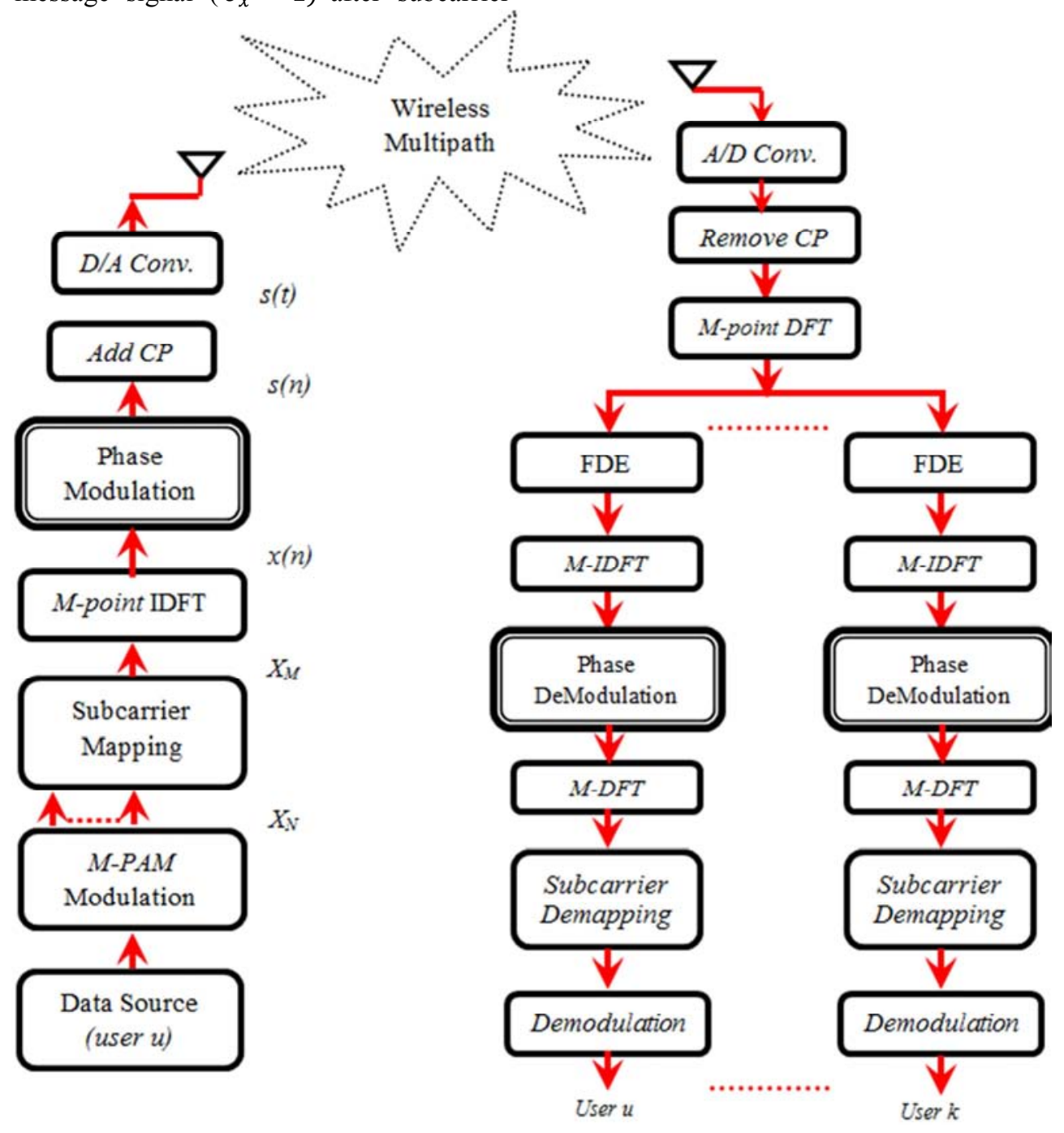

Figure 2. A block diagram for the proposed DFT-OFDMA-PM Transceiver system.

$X_{k}$ is the resulted sequence after subcarrier mapping and $q_{k}$ $(t)$ are the orthogonal subcarriers which are also must be realvalued for phase modulation $[5,13]$,

$$
q_{k}(t)=\cos \left(\frac{\pi k t}{\mathrm{~T}}\right)+\sin \left(\frac{2 \pi k t}{T}\right), 0 \leq t<T, 1 \leq k<M
$$

From Eqs.(1) and (2), the phase signal, $\varnothing(t)$, can be written as follows:

$$
\emptyset(t)=\theta+2 \pi h C_{N} \sum_{k=1}^{M} X_{k} q_{k}(t)
$$

The received signal is.

$$
r(t)=\int_{0}^{\tau_{\max }} h(\tau, t) s(t-\tau) d \tau+z(t)=\int_{0}^{\tau_{\max }} h(\tau) s(t-\tau) d \tau+z(t)
$$

where $h(\tau, t)$ is the channel impulse response (CIR), $\tau_{\max }$ is the channel's maximum propagation delay, and $z(t)$ is the additive white Gaussian noise. The channel is assumed to be static over the block interval, and therefore, $h(\tau, t)=h(\tau)$.

The addition of the CP makes the linear convolution with the CIR equivalent to a circular convolution. Thus, by choosing $N_{g} \geq L$ ( $L$ is the length of the CIR), the received samples, $r(n)$ are represented equivalently by a linear $(*)$ or a circular ( ) convolution,

$$
r_{n}=s_{n} * h_{n}+z_{n}=\left(s_{n} * h_{n}\right)_{c i r}+z_{n}=\operatorname{IDFT}\left\{S_{k} H_{k\}+z_{n}}\right.
$$


where $\left\{S_{k}, H_{k}\right\}$ are the DFTs of $\left\{s_{n}, h_{n}\right\}$ respectively. Then, after the $\mathrm{A} / \mathrm{D}$ converter, the $\mathrm{CP}$ samples are discarded and the remaining samples are equalized by frequency domain equalization (FDE). The FDE can perfectly invert the effect of the channel. Its advantage is the relatively low complexity, and the disadvantage is the requirement of a $\mathrm{CP}$ overhead. The block of FDE includes three blocks, DFT, FDE, and IDFT respectively and is shown in Figure 2. The FDE output is:

$$
\hat{s}_{n}=\operatorname{IDFT}\left\{R_{k} C_{k}\right\}=\left(r_{n} * c_{n}\right)_{\text {cir }}
$$

where $R_{k}$ is the DFT of the received signal $r_{n}$ and $C_{k}$ represents the equalizer correction term, which is computed according to the type of the equalizer. For MMSE equalizer the correction term is computed as follows [15-18]:

$$
C_{k}=\frac{H_{k}^{*}}{\left|H_{k}\right|^{2}+\left(S N R^{-1}\right)}
$$

where $S N R$ is the signal-to-noise ratio. After that, the time domain signal is applied to the phase demodulator as shown in Figure 3.

The phase demodulator includes three operations, a finite impulse response (FIR) filter which is used to improve the phase demodulator performance, the arg (.) is used to extract the phase of the signal. Finally, the phase unwrapper is used to reduce the effect of phase ambiguities and makes the receiver insensitive to phase offsets caused by the channel and the memory term.

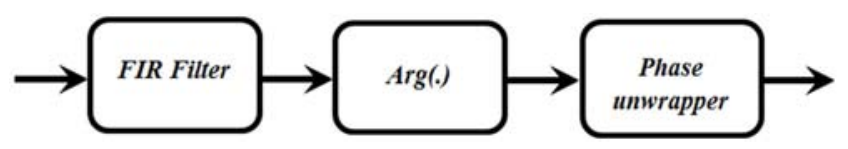

Figure 3. Phase demodulator.

\subsection{The DCT-OFDMA-PM System}

This section presents the main difference of the recently $p$ roposed system, the DCT-OFDMA-PM, introduced by F. Alfuhaidy to our proposed DFT-OFDMA-PM system [5]. By R eferring to the block diagram of the DCT-OFDMA-PM, it sh ows high similarity to our proposed system shown in Figure
2, except the uses of IDCT at the transmitter and the DCT at $t$ he receiver, which is a single sinusoidal as presented in Eq.(1 0 ) rather than a combination of sinusoidal and cosinusoidal $f$ unctions of our proposed system shown by above subsection in Eq.(4).

$$
q_{k}(t)=\cos \left(\frac{\pi k t}{T}\right), 0 \leq t<T, 1 \leq k<M
$$

Moreover, the performance is analyzed and compared by this paper for the two systems using simulation which illustra tes the improvement introduced by the proposed system.

\section{Simulations and Results}

This section presents and discusses simulation results for the proposed system, the DFT-OFDMA-PM, and compares it to the DCT-OFDMA-PM system for different subcarrier techniques, localized and interleaved mapping. Moreover, the modulation index, $h$, is studied and the optimum value is chosen using simulation. We note that the abbreviations DFT-LOFDMA-PM and DFT-IOFDMA-PM refer to localized and interleaved subcarrier mapping respectively. Similarly, DCT-LOFDMA-PM and DCT-IOFDMA-PM for DCT-based system localized and interleaved subcarrier mapping respectively.

\subsection{Simulation Parameters}

Since that the DFT-OFDMA-PM system was not introduced by $\mathrm{F}$. Al-fuhaidy or other previous researches, it will be introduced by this paper [5]. Although, and for comparison purpose, the DCT-OFDMA-PM which is recently proposed by $\mathrm{F}$. Al-fuhaidy is also simulated [5]. A Monte Carlo simulation with $10^{4}$ iterations for each scenario is used to evaluate the performance of different systems.

For simulation of the DFT-OFDMA-PM, and the DCTOFDMA-PM systems, simulation parameters are chosen similarly to parameters used by different previous researches that simulate the Constant-Envelope-OFDM with some additional special parameters used for OFDMA as shown in Table $1[5,9,13,16]$.

Table 1. Simulation parameters for DFT-OFDMA-PM, and DCT-OFDMA-PM systems.

\begin{tabular}{lll}
\hline & Description & Parameters \\
\hline & Block size for each user ' $N$ ' & 64 \\
& Modulation & 4 -PAM \\
& Number of users ' $Q$ ' & 4 \\
Transmitter & Modulation index ' $h$ ' & $0.0 / 2 \pi$, to $2.0 / 2 \pi$, step by 0.2 \\
& Oversampling ' $J$ ' & 8 \\
& $D C T$ ' $N_{D C T}$ ' and $D F T$ size, ' $N_{D F T}{ }^{\prime}$ & $N^{*} Q * J=2048$ \\
& Block period ' $T$ ' & $128 \mu$ s \\
& Subcarrier mapping & Localized and Interleaved \\
Channel & Channel model & Vehicular A outdoor channel \\
& Noise environment & AWGN \\
Receiver & Channel estimation & Perfect channel \\
& Equalization & MMSE \\
\hline
\end{tabular}




\subsection{Performance of the Proposed DFT-OFDMA-PM System}

This subsection presents simulation results for the proposed system, the DFT-OFDMA-PM. The performance of the proposed system is discussed and analyzed in terms of the BER, the modulation index, and the PAPR, and compared to the DCT-OFDMA-PM system and the traditional system.

\subsubsection{The Selection of the Optimum Modulation Index}

A well-known problem in phase modulation scheme is the nonlinearity for the spectrum transformation of the phase modulated signal. However, by proper choice of the modulation index $h$, this problem is elevated. An investigation of the effect of the modulation index, $h$, on the BER performance is carried via simulation results analysis of the proposed system for different values of, $h$. Moreover, and in order to verify this analysis, this study extended by involving the DCT-OFDMA-PM system simulation results. Actually, it is noted that the performance of the proposed system is degraded for some values of $h$. Figures $4,5,6$, and 7 show different curves for the $B E R$ versus $E_{b} / N_{0}$ of the proposed DFT-OFDMA-PM and the DCT-OFDMA-PM systems with different values, $0.2 / 2 \pi, 1.0 / 2 \pi$, and $1.4 / 2 \pi$, of modulation index, $h$.

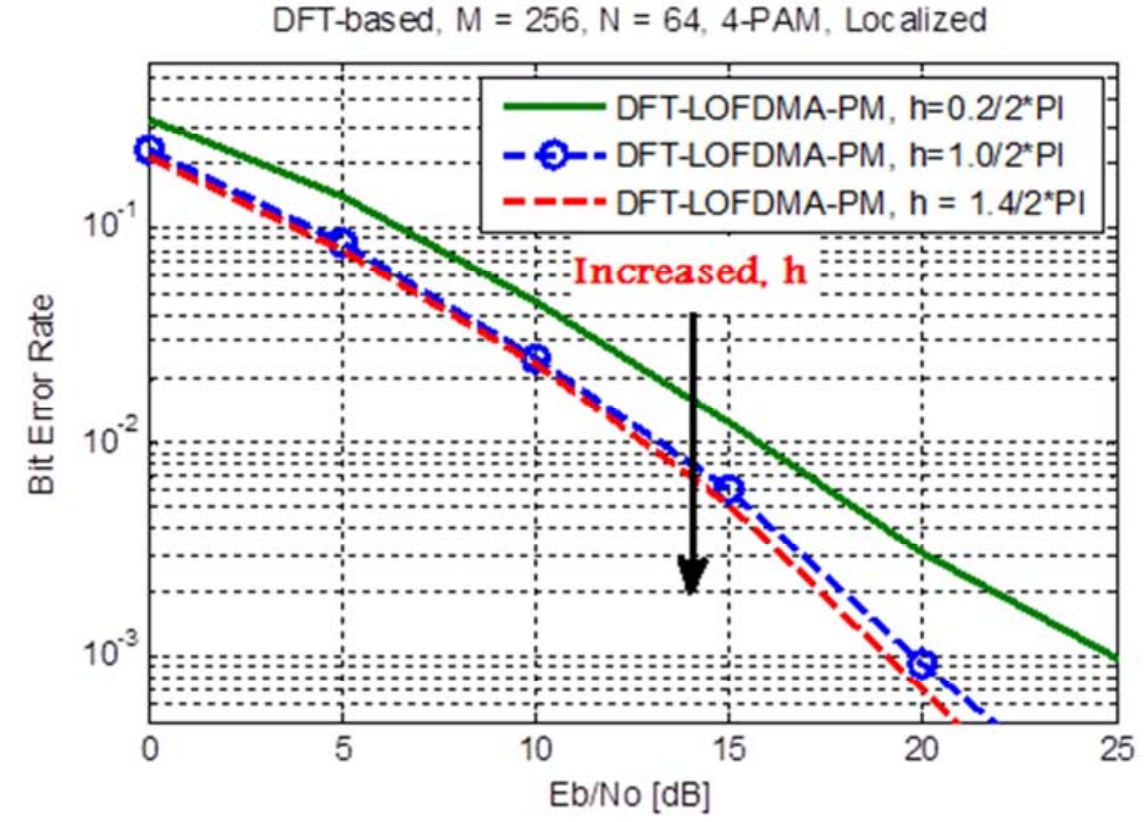

Figure 4. BER vs. $E_{b} / N_{0}$ for DFT-LOFDMA-PM and different modulation index, $h$.

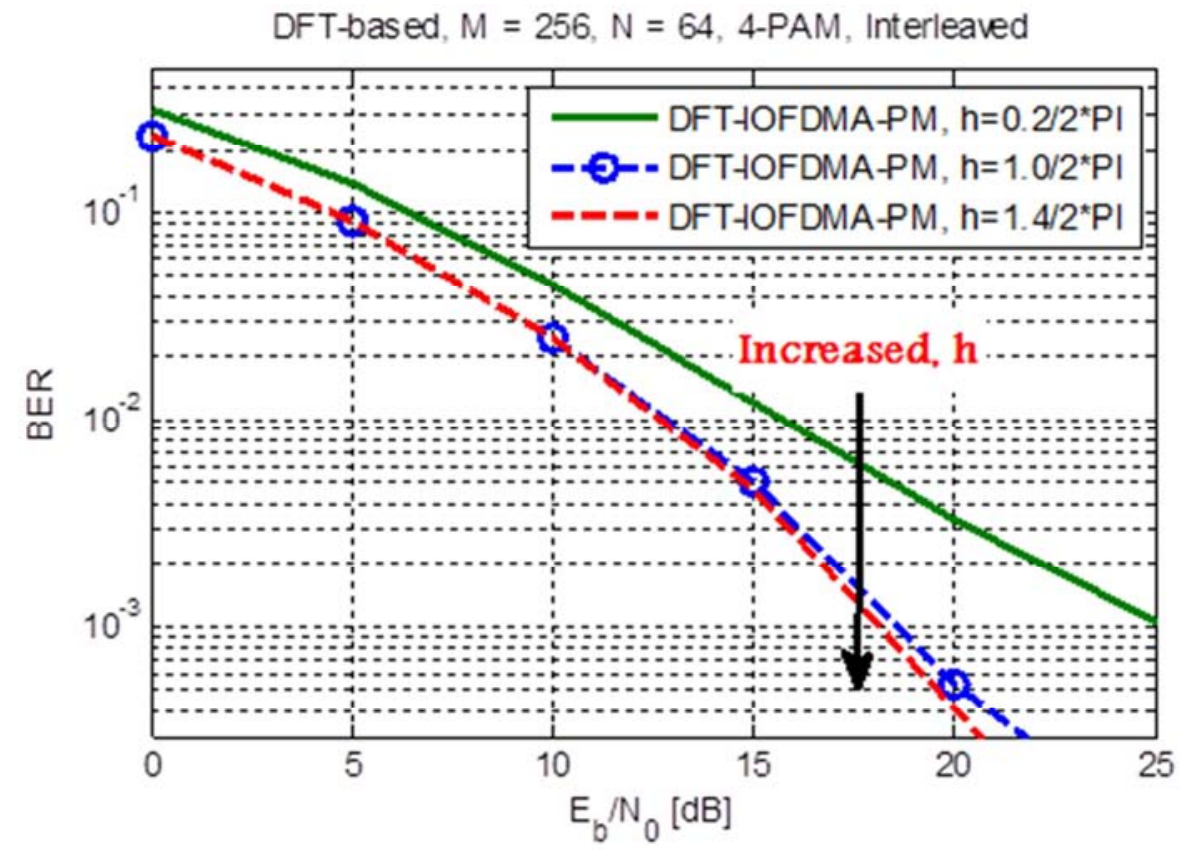

Figure 5. BER vs. $E_{b} / N_{0}$ for DFT-IOFDMA-PM and different modulation index, $h$. 




Figure 6. BER vs. $E_{b} / N_{0}$ for DCT-LOFDMA-PM and different modulation index, $h$.

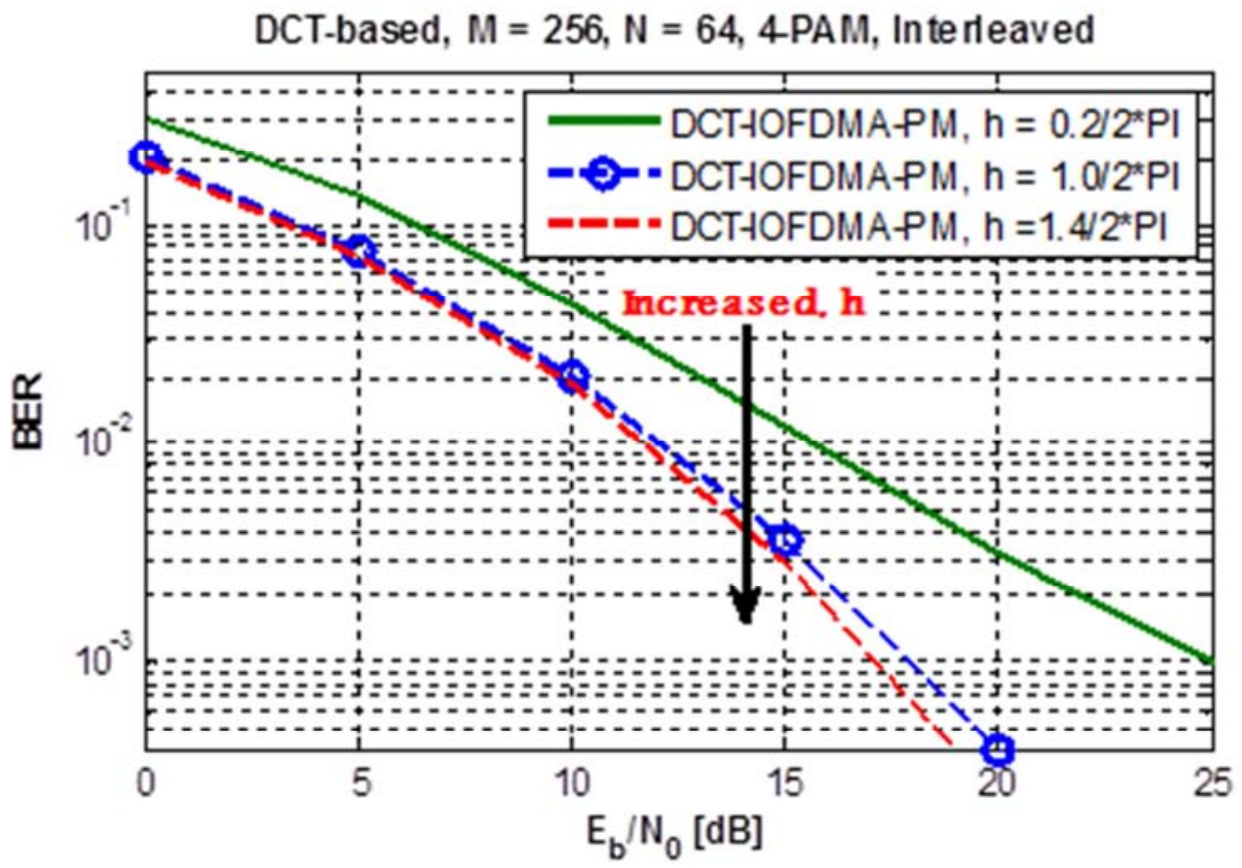

Figure 7. BER vs. $E_{b} / N_{0}$ for DCT-IOFDMA-PM and different modulation index, $h$.

It is clear from the above figures that BER performance is decreased as $h$ increased. Also, it is noticed that for $h$ greater than $1 / 2 \pi$, the BER may has a small improvement at $E_{b} / N_{0}$ equal or greater than $20 \mathrm{~dB}$ while, the $E_{b} / N_{0}$ is the dominant.

In this paper, the optimum value of $h$ is selected to be equal to $1.0 / 2 \pi$. This selection takes in consideration that the modulation index controls the system performance bandwidth metric. A useful bandwidth expression for the continuous PM signal is the root-mean-square (RMS) bandwidth $[9,13]$ :

$$
B W=\max (2 \pi h, 1) W
$$

As shown in Eq. (11), the signal bandwidth grows with $2 \pi h$, which in turn reduces the bandwidth efficiency. Exhaustive simulation scenarios for the BER against $E_{b} / N_{0}$ and for wide range values of $h$ are carried and presented by the following figures. Figures 8 and 9 demonstrate variations of the BER for different signal to noise ratio and different modulation index. A clear indication about modulation index is shown. The BER decreases as modulation index increased from $0.0 / 2 \pi$, up to $1.0 / 2 \pi$ step by 0.2 , after that the BER 
either has small decrease with localized subcarrier mapping or it increases with interleaved subcarrier mapping. Thus, the modulation index, $h=1.0 / 2 \pi$, can be selected as an optimum value for good BER and saved system bandwidth.

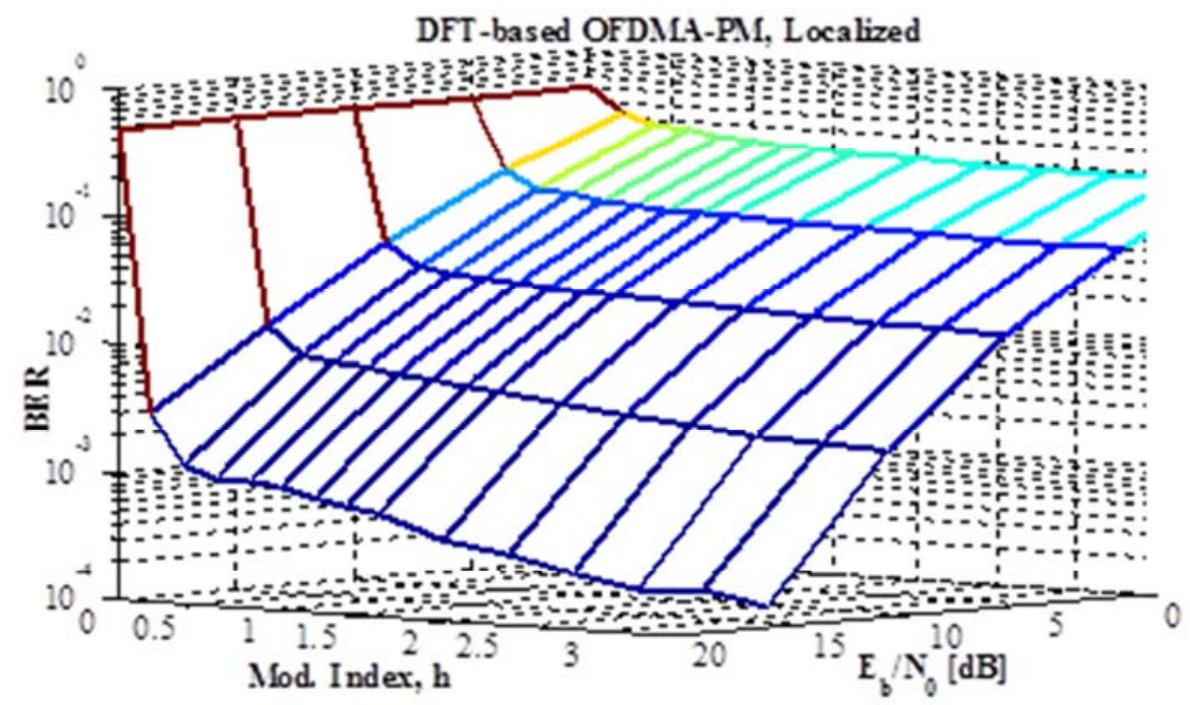

Figure 8. Variation of the BER with modulation index, $h$, and $E_{b} / N_{0}$ for the proposed DFT-LOFDMA-PM.

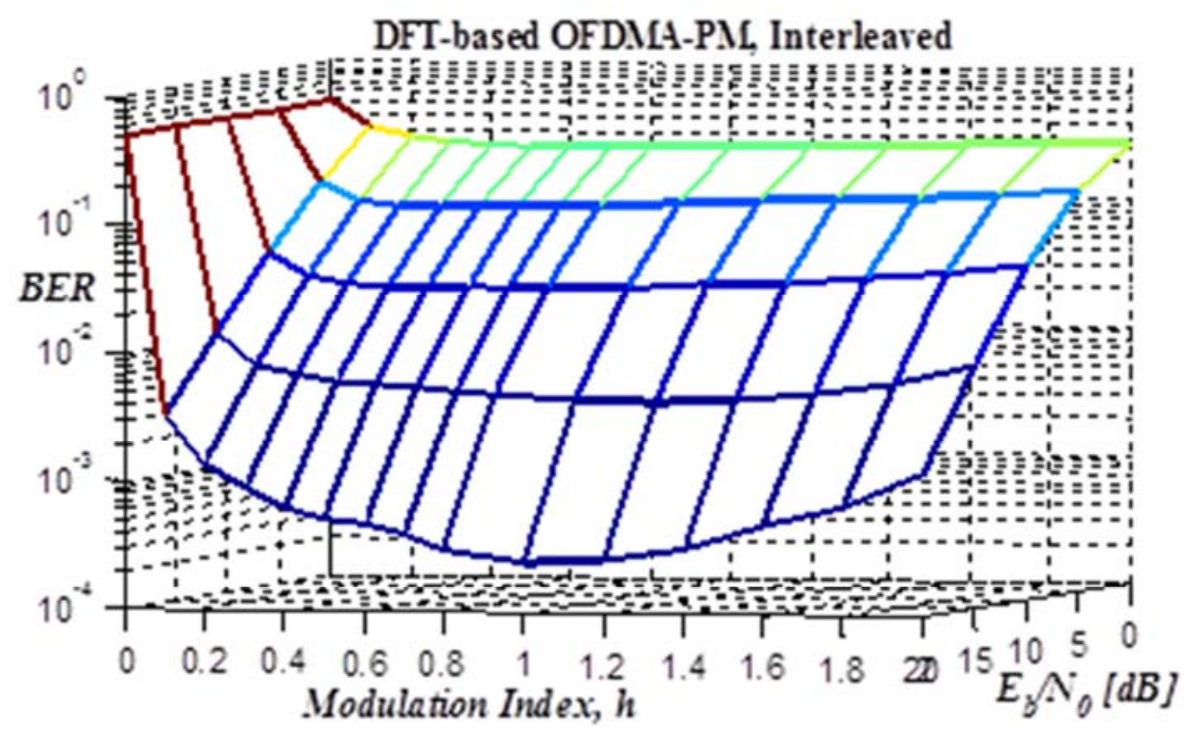

Figure 9. Variation of the BER with modulation index, $h$, and $E_{b} / N_{0}$ for the proposed DFT-IOFDMA-PM.

\subsubsection{BER Improved Performance Introduced by the Proposed System in Term of BER}

In this subsection, the BER performance of the proposed system, the DFT-OFDMA-PM, is compared to the BER performance of the DCT-OFDMA-PM and the conventional DFT-based and DCT-based OFDMA systems. The modulation index, $h$, is selected to be equal to the optimum value, $1.0 / 2 \pi$ Fig. 10 and 11 illustrates the BER performance of the proposed system, the DFT-OFDMA-PM when compared to the DCT-OFDMA-PM system, and to the DFTbased and the DCT-based systems without PM, for different subcarrier mapping, localized and interleaved respectively. It is also clear from figures that the DFT-OFDMA-PM has small improvement in BER when compared to DCTOFDMA-PM in case of localized subcarrier mapping and vice versa in case of interleaved subcarrier mapping. At this point, we shall note that the localized subcarrier mapping is the preferred choice in broadband communication system. This is due to many problems associated with interleaved subcarrier mapping such as carrier frequency offset. By looking to the improvement introduced by the proposed DSTOFDMA-PM system when compared to the conventional systems. The proposed DST-LOFDMA-PM provides about $4.5 \mathrm{~dB}$ and $3 \mathrm{~dB} E_{b} / N_{0}$ enhancement at $B E R=10^{-2}$ when compared to DFT-based and DCT-based systems without PM respectively. Approximately, similar improvement achieved by the DFT-IOFDMA system compared to the conventional systems without PM.

We note that the improvement introduced by the proposed system is due to the ability of the phase modulation to exploit the diversity of multipath channels. 


\subsubsection{The PAPR Performance}

This section presents simulation results for the proposed system in term of PAPR when compared to the conventional system. Figures 12 and 13 present the PAPR for the DFTOFDMA-PM and the traditional OFDMA system, with localized and interleaved mapping respectively. It is clear from figures, that the proposed system provides the better PAPR, $0 \mathrm{~dB}$, while the conventional system posed from a very high PAPR. Intuitively, this is due to that the proposed system transmit a constant envelop fluctuated signal, along with the information signal is phase modulated. This improvement clears the effect of the PAPR problem in the proposed system which was considered as a main challenge in $4 \mathrm{G}$ mobile generation.

This improvement in case of constant envelop transmitted signal, neglect the effect of the power amplifier of the transmitted device, the mobile terminal. And lower the cost by using nonlinear power amplifier device with the mobile terminal, uplink transmission. We note that this challenge is not considered by the downlink transmission, the base station.

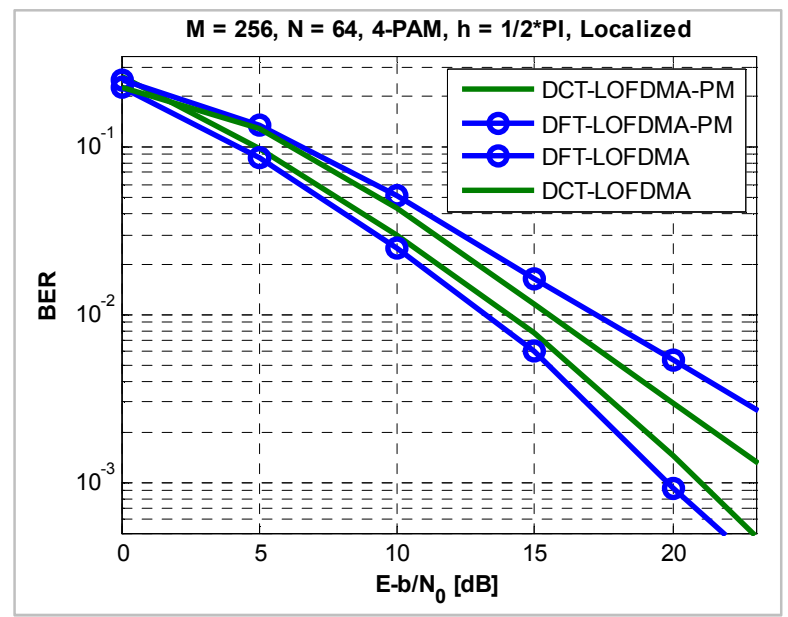

Figure 10. BER vs. $E_{b} / N_{0}$ for DFT-LOFDMA-PM and different systems.

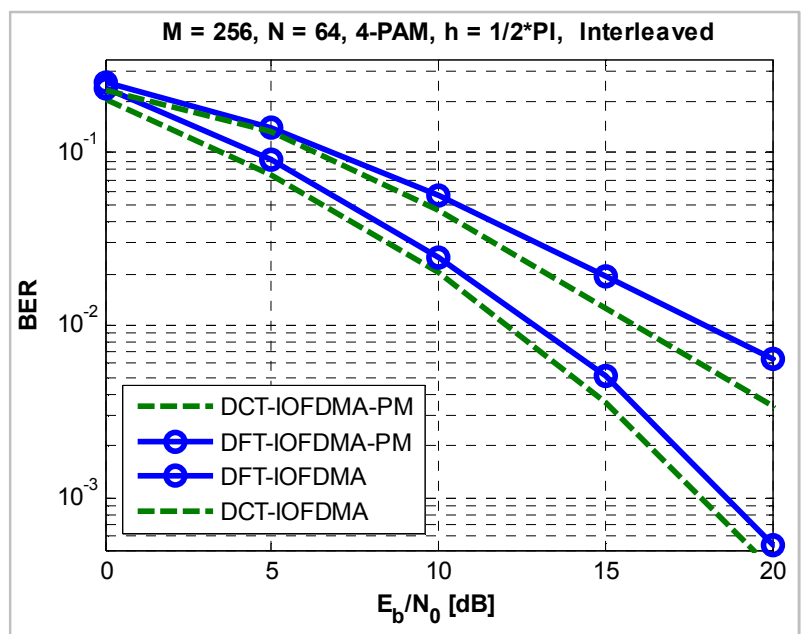

Figure 11. BER vs. $E_{b} / N_{0}$ for DFT-IOFDMA-PM and different systems.

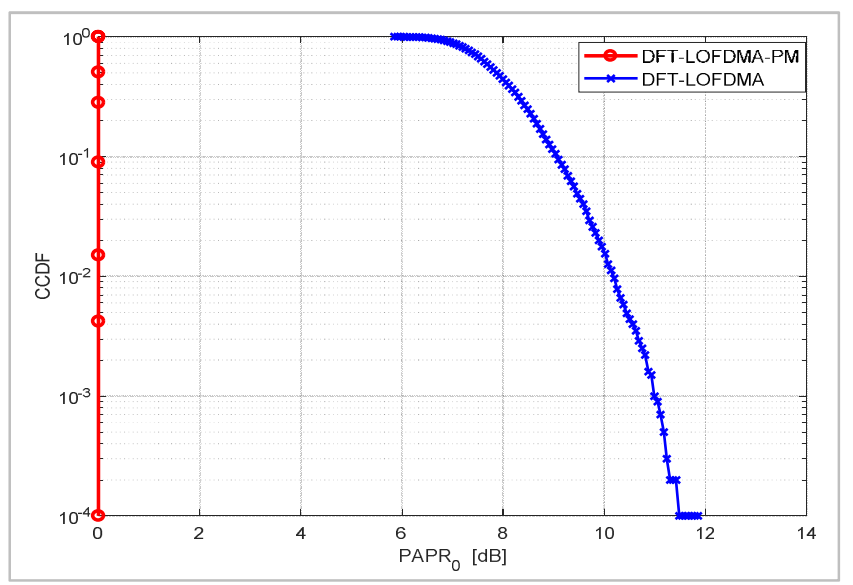

Figure 12. CCDF vs.PAPR for the DFT-LOFDMA-PM and the conventional OFDMA system.

\section{Conclusions}

This paper was proposed and investigated using simulation a new transceiver scheme for the OFDMA system by adding phase modulation to the resulted signal before transmission. This system was called DFT-OFDMA-PM. The proposed system has different advantages such as reduced PAPR and improved BER performance, which are the common problems in the broadband communications. The proposed DFT-OFDMA-PM system provides a constant envelope signal, i.e., OdB PAPR, due to constant amplitude output signal since the transmitted signal is phase modulated. This is the main advantage of the proposed system which play as a main challenge in broadband communication either in $3 \mathrm{G}$ or 4G mobile communication. Moreover, the proposed system improved the BER performance. As shown by simulation results, the proposed system provide a significant enhancement in BER when compared to the conventional systems and the recently proposed DCT-OFDMA-PM system which is desired in broadband communications. Finally, since, the proposed system add a PM block to the conventional system which affected by the modulation index so, the effect of the modulation index was also studied by executing exhaustive simulation scenarios and studying its effect on performance, and the optimum value was selected. Simulation results show the effectiveness of the proposed system in broadband communications.

\section{References}

[1] 3rd Generation Partnership Project (3GPP) Technical Specification Group Radio Access Network; Physical Layer Aspects for Evolved Universal Terrestrial Radio Access (UTRA) (Release 7). 3GPP TR 25. 814, V7. 1. 0, Sept 2006.

[2] FALCONER D, ARIYAVISITAKUL S. L, BENYAMINSEEYAR A, and EIDSON B, "Frequency domain equalization for single-carrier broadband wireless systems", IEEE Commun. Mag., 2002, 40, pp. 58-66. 
[3] ADACHI F., GARGE D., TAKAOKA S., TAKEDA K.: "Broadband CDMA techniques", IEEE Wirel. Comm., 2005, $12,(2)$, pp. $8-18$.

[4] Air Interface for Fixed and Mobile Broadband Wireless Access Systems Amendment for Physical and Medium Access Control Layers for Combined Fixed and Mobile Operation in Licensed Bands, IEEE Std 802. 16e, 2006.

[5] Farouk A. K. Al-fuhaidy, Hossam Eldin A. Hassan, and Khairy El-barbary, "A New Transceiver Scheme for OFDMA System Based on Discrete Cosine Transform and Phase Modulations", WPC Springer Journal, 2012.

[6] S. H. Han and J. H. Lee, "An Overview of Peak-to-Average Power Ratio Reduction Techniques for Multicarrier Transmission," IEEE Wireless Communications, vol. 12, no. 2, 2005, pp. 56-65.

[7] J. Armstrong, "Peak-to-Average Power Reduction for OFDM by Repeated Clipping and Frequency Domain Filtering," Electron. Lett., vol. 38, Feb. 2002, pp. 246-247.

[8] Seng-Hung Wang, Jia-Cheng Xie and Shih-Peng Li, "A LowComplexity SLM PAPR Reduction Scheme for Interleaved OFDMA Uplink," IEEE GLOBECOM, 2009.

[9] S. C. Thompson, A. U. Ahmed, J. G. Proakis, J. R. Zeidler, and M. J. Geile "Constant Envelope OFDM," IEEE TRANSACTIONS ON COMMUNICATIONS, VOL. 56, NO. 8, Aug 2008.

[10] S. C. Thompson, A. U. Ahmed, J. G. Proakis, and J. R. Zeidler, "Constant Envelope OFDM Phase Modulation: Spectral Containment, Signal Space Properties and Performance," in Proc. IEEE Milcom, vol. 2, Monterey, pp. 1129-1135, Oct. 2004.

[11] J. G. Proakis and M. Salehi, "Communication Systems Engineering,” New Jersey: Prentice Hall, 1994.

[12] Emad S. M. Hassan, "Performance Enhancement of MultipleInput Multiple-Output OFDM-Based Wireless Systems," PhD Thesis, Menofia University, 2010.

[13] R. Nogueroles, M. Bossert, A. Donder, and V. Zyablov "Improved Performance of a Random OFDMA Mobile Communication System," proceeding of the IEEE VTC, Vol. 3, pp. 2502-2506, May 1998.

[14] H. G. Myung, J. Lim and D. J. Goodman, "Single Carrier FDMA for Uplink Wireless transmission," Proceeding of the IEEE Vehicular Technology Magazine., vol. 1, no. 3, 2006.

[15] F. S. Al-kamali, M. I. Dessouky, B. M. Sallam, F. Shawki and F. E. Abd El-Samie, "Tranceiver Scheme for Single-Carrier Frequency Division Multiple Access Implementing the Wavelet Transform and Peak-to-Average power Ratio reduction methods," IET Comm, 2010, Vol 4, Iss. 1, pp. 69-79.

[16] F. S. AL-Kamali, “A New Single-Carrier Transceiver Scheme Based On The Discrete Sine Transform”, JOE, 2014.
[17] F. S. Al-kamali, F. A. Al-Fuhaidy, and K. A. Al-soufy, "Wireless Image Transmission Over Frequency Selective Channel Using Recent OFDMA Systems," AJCCC, 2018.

[18] K. M. Al-soufy, F. S. Al-kamali, and F. A. Al-Fuhaidy, "Performance Evaluation of SC-FDMA Systems Using Wireless Images," AJCSA, 2017.

\section{Biography}

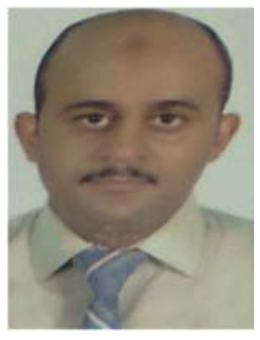

Farouk Abduh Kamil Al-Fuhaidy. He received the B. S. E. E. Computer Engineering and M. S. E. E. in Computer Engineering respectively and $\mathrm{Ph}$. D. in Communication Engineering from the Military Technical College, Cairo, Egypt in 2007, 2009 and 2013 respectively. He joined the teaching staff of the Department of Electrical Engineering, Faculty of Engineering and Architecture, Ibb University, Yemen, in 2014. His research interests and activities are in the areas of CDMA, OFDMA, SC-FDMA Systems, Wireless Sensor Networks and adhoc Networks Routing Protocols, Embedded Systems, IOT, broadband communications, and advanced signal and image processing Techniques.

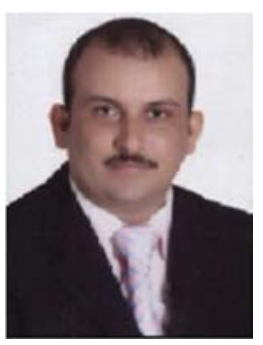

Faisal Saif Alkamali has received the B. Sc. degree in Electronics and Communications Engineering from Baghdad University, Baghdad, Iraq, in 2001. He has received the M. Sc., and $\mathrm{PhD}$ degrees in Communication Engineering from Menoufia University, Menouf, Egypt, in 2008, and 2011, respectively. He joined the teaching staff of the Department of Electrical Engineering at Ibb University, Ibb, Yemen in 2011. He has served as a head of the Electrical department, from Oct. 2013 to Oct. 2014. His research areas of interest include CDMA, OFDMA, SC-FDMA Systems, MIMO Systems, Interference Cancellation, Synchronization, and Channel Equalization \& Estimation.

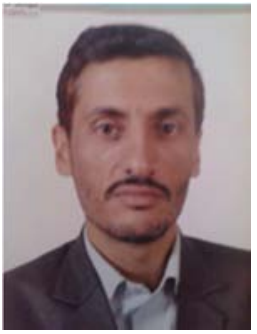

Khaled Al-Soufy. He received his B. Sc in Computer and control engineering from Sanaa University, Yemen, in 1998. He received his M. Tech in Computer Science and Engineering from Osmania University, Hyderabad, India in 2006. He received his $\mathrm{Ph}$. D. in 2015 in Computer Engineering, from Z. H. College of Engineering and Technology, Aligarh Muslim University, Aligarh, India. He has joined the teaching staff of the Department of Electrical Engineering, Faculty of Engineering and Architecture, Ibb University, Yemen, in 2013. His research interest includes mobile computing and QoS in mobile ad hoc \& WSNs networks, and image and signal processing. 